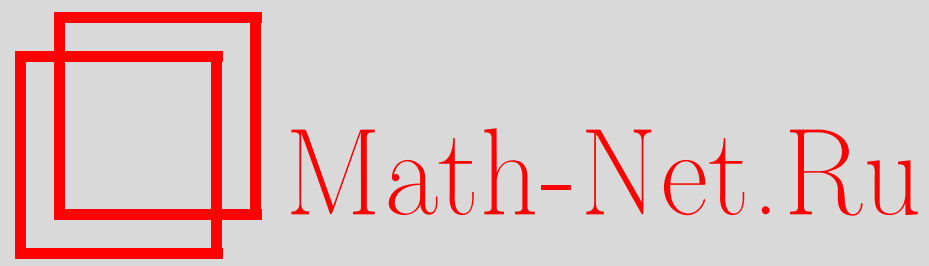

А. Т. Ильичев, Уединенные волны в холодной плазме, Матем. заметки, 1996, том 59, выпуск 5, 719-728

DOI: https://doi.org/10.4213/mzm1766

Использование Общероссийского математического портала MathNet.Ru подразумевает, что вы прочитали и согласны с пользовательским соглашением

http://www. mathnet.ru/rus/agreement

Параметры загрузки:

IP : 44.207 .124 .84

26 апреля 2023 г., 13:37:20

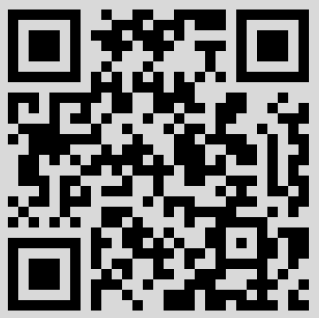




\section{УЕДИНЕННЫЕ ВОЛНЫ В ХОЛОДНОЙ ПЛАЗМЕ}

\section{А. Т. Ильичев}

Введение. Понижение порядка динамической системы методом редукции на центральное многообразие (см. [1], [2]) является плодотворньм методом решения задач механики и физики, где требуется определить формы бегуших волн, являюшихся бифуркацией из состояния покоя, т.е. тривиального решения рассматриваемой динамической системы. Поток на центральном многообразии описьвает все ограниченные решения, не покидающие малой окрестности состояния покоя для всех положительньх и отрицательных значений “времени". Роль “времени" для бегуших волн играет пространственная переменная $-\infty<\xi<\infty$. Все малые решения динамической системы

$$
\dot{\mathbf{w}}=A \mathbf{w}+F(\mu, \mathbf{w})
$$

$\left(A: \mathbb{R}^{n} \rightarrow \mathbb{R}^{n}\right.$ - линейный оператор (постоянная матрица), среди собственных значений которой находятся чисто мнимые, $F \in C^{k}\left(\mathbb{R}^{n}, \mathbb{R}^{n}\right)$ для достаточно большого целого $k, F(0,0)=0, \partial_{\mathbf{w}} F(0,0)=0, \mu$ - параметр бифуркации) лежат на центральном многообразии $\mathbf{w}_{1}=h\left(\mu, \mathbf{w}_{0}\right)$, $\mathbf{w}_{0} \in E_{0}$-инвариантному подпространству, натянутому на собственные векторы, отвечающие чисто мнимым собственным значениям $A, \mathbf{w}_{1} \in E_{h}$, $\mathbb{R}^{n}=E_{0} \oplus E_{h}$ и $\mathbf{w}=\mathbf{w}_{0}+\mathbf{w}_{1}$. Функция $h$ имеет второй порядок малости по $\mu$ и $\mathbf{w}_{0}$ и наследует свойства симметрии системы (1).

Отметим, что в последнее время получил распространение метод редукции для систем типа (1) в гильбертовых пространствах, в частности, широкий интерес вызьвают модельные задачи, связанные с редукцией уравнений в частных производных в цилиндрических областях. Если инвариантное подпространство, отвечающее мнимым собственным значениям оператора $A$ конечномерно, то уравнение в частных производных, где неограниченная пространственная переменная трактуется как "время", при определенных свойствах резольвенты оператора $A$ сводится к конечномерной динамической системе. Развитие метода редукции для динамических

Работа выполнена при поддержке Российского фонда фундаментальных исследований, грант № 93-013-17355. 
систем в гильбертовых пространствах было впервые предпринято в [3] и получило свое завершение (для квазилинейных систем) в [4]. Среди работ, где рассматривается приложение обсуждаемого метода к модельным задачам механики и физики, отметим работу [5], где изучается распространение нелинейных волн в упругой полосе, а также [6], где описьваются все ветви нелинейных бегущих волн, являющиеся бифуркацией из состояния покоя, в идеальной жидкости конечной глубины с учетом капиллярных эффектов.

В настоящей работе исследуется вопрос наличия уединенных волн в холодной плазме. Уравнения, описывающие плоские бегущие волны в холодной плазме, достаточно сложны, вследствие чего известно лишш несколько частных решений солитонного типа [7]. В работах [8], [9] для длинных слабонелинейых волн формально были получены уравнения Кортевега-де Вриза для $\varphi \neq \varphi_{c}$ (где $\varphi$ - угол между вектором магнитного поля в состоянии покоя и направлением движения, а $\varphi_{c}-$ критическое значение этого угла) и уравнение с пятой производной для $\varphi=\varphi_{c}$. Формальньй вьвод при $\varphi$ близких к $\varphi_{c}$ дает эволюционное уравнение с квадратичной нелинейностью и третьей и пятой производной. Для этого модельного уравнения (в случае разных знаков при третьей и пятой производной) в работах [10], [11] было доказано существование уединенных волн, а также установлена их нелинейная устойчивость.

В настоящей работе исследуется полная система уравнений холодной плазмы на предмет существования семейства уединенных волн, зависящего от параметра. Следуя [6], выделим три последовательных этапа этого исследования:

- сведение динамической системы (4) четвертого порядка к системе второго порядка на центральном многообразии (п. 1);

- приближение системы на центральном многообразии последовательностью интегрируемых систем, описание всех ограниченных решений аппроксимирующей системы (п. 2);

- доказательство грубости солитоноподобных решений по отношению к любьм обратимьм возмущениям высшего порядка по амплитуде (п. 3), обратимость этих возмущений отвечает соответствующей симметрии исходных уравнений.

Доказательство существования солитоноподобных решений, таким образом, представляет собой комбинацию асимптотических методов и приемов, связанных с доказательством “выживания" решений указанного типа для полной системы уравнений. Применение асимптотических методов (типа ВКБ) в теории плазмы с малой нелинейностью, описьваемой МГД уравнениями, хорошо известно (см., например, [12]). В рассматриваемом случае искомая система приближается интегрируемьпи уравнениями в квази-нормальной форме. Для доказательства грубости уединенных волн для полной системы уравнений, как правило, используются подходящие 
версии теоремы о неявной функции. В данном случаеприменяется теорема о неявной функции для оператора, действуюшего в банаховом пространстве экспоненциально убывающих функций.

1. Одномерные движения холодной квазинейтральной плазмы описываются системой уравнений [8]

$$
\begin{gathered}
\frac{\partial n}{\partial t}+\frac{\partial(n u)}{\partial x}=0, \quad \frac{d u}{d t}+n^{-1} \frac{\partial}{\partial x}\left[\frac{B_{y}^{2}+B_{z}^{2}}{2}\right]=0, \\
\frac{d v}{d t}-n^{-1} B_{x} \frac{\partial B_{y}}{\partial x}=-R_{e}^{-1} \frac{d}{d t}\left(n^{-1} \frac{\partial B_{z}}{\partial x}\right), \\
\frac{d w}{d t}-n^{-1} B_{x} \frac{\partial B_{z}}{\partial x}=R_{e}^{-1} \frac{d}{d t}\left(n^{-1} \frac{\partial B_{y}}{\partial x}\right), \\
\frac{d B_{y}}{d t}-B_{x} \frac{\partial v}{\partial x}+B_{y} \frac{\partial u}{\partial x}=R_{i}^{-1} \frac{\partial}{\partial x}\left(\frac{d w}{d t}\right) \\
\frac{d B_{z}}{d t}-B_{x} \frac{\partial w}{\partial x}+B_{z} \frac{\partial u}{\partial x}=-R_{i}^{-1} \frac{\partial}{\partial x}\left(\frac{d v}{d t}\right)
\end{gathered}
$$

$(d / d t \equiv \partial / \partial t+u \partial / \partial x)$, где независимая переменная $x$, плотность числа частиц $n$, напряженность магнитного поля $\mathbf{B}=\left(B_{x}, B_{y}, B_{z}\right)$ и скорость ионов $\mathbf{v}=(u, v, w)$ обезразмерены при помоши характерной длины $l$, плотности невозмущенной плазмы $n_{0}$, невозмущенного магнитного поля $\mathbf{B}_{0}$ и альфвеновской скорости $V_{A}=\left|\mathbf{B}_{0}\right| / \sqrt{4 \pi n_{0}\left(m_{i}+m_{e}\right)}, m_{i}, m_{e}-$ соответственно массы иона и электрона. Параметры дисперсии $R_{i}$ и $R_{e}$ даются формулами $R_{i}=\omega_{i c} / \omega_{0}, R_{e}=\omega_{e c} / \omega_{0}$, где $\omega_{i c}$ - ионная, $\omega_{e c}-$ электронная циклотронные частоты, а $\omega_{0}=V_{A} / l$ - характерная частота явления. Одномерные движения холодной плазмы характеризуются тем, что компонента $B_{x}$ вектора напряженности магнитного поля остается постоянной в течение всего времени движения. Переменные $n, u, v, w$, $B_{x}, B_{y}, B_{z}$ в состоянии покоя имеют значения $1,0,0,0, \cos \varphi, \sin \varphi, 0$ соответственно. Здесь $\varphi$ - угол наклона вектора магнитного поля к направлению движения, совпадаюшему с осью $x$. Дисперсионная кривая в данном случае состоит из двух ветвей и при малых волновых числах $k$ (больших длинах волн) фазовые скорости $V_{\Phi}^{ \pm}(k)$ имеют вид [8], [9]

$$
\begin{aligned}
& V_{\Phi}^{+}=1-\frac{1}{2 R_{i} R_{e}}\left\{1-\left(\sqrt{\frac{R_{e}}{R_{i}}}-\sqrt{\frac{R_{i}}{R_{e}}}\right)^{2} \operatorname{ctg}^{2} \varphi\right\} k^{2}+\cdots, \\
& V_{\Phi}^{-}=\cos \varphi\left[1-\frac{1}{2 R_{i} R_{e}}\left\{1+\left(\sqrt{\frac{R_{e}}{R_{i}}}-\sqrt{\frac{R_{i}}{R_{e}}}\right)^{2} \operatorname{ctg}^{2} \varphi\right\} k^{2}+\cdots\right] .
\end{aligned}
$$

Скорость $V_{\Phi}^{+}(k)$ соответствует магнитозвуковой волне, а $V_{\Phi}^{-}(k)$ - альфвеновской волне. В пределе $k \rightarrow 0$ из (3) получим $V_{\Phi}^{+}=V_{\Phi}^{+}(0)=1$, 
$V_{\Phi}^{-}=V_{\Phi}^{-}(0)=\cos \varphi$. Далее будут рассматриваться бегущие волны, скорость которых мало отличается от скорости магнитозвуковых волн бесконечной длины, т.е. нас будут интересовать решения уравнений (2) типа бегушей волны, распространяюшейся со скоростью $V=1+\mu / 2$, где $\mu-$ малый параметр. Решения уравнений (2), соответствуюшие бегушим волнам, зависят только от $\xi=x-V t$ и описываются системой, полученной однократньм интегрированием из (2). При этом плотность $n$ и $x$-компонента скорости алгебраически зависят от остальных неизвестных функций:

$$
\begin{gathered}
n^{-1}=1-\frac{1}{2 V^{2}}\left(\widehat{B}_{y}^{2}+2_{0} B_{y} \widehat{B}_{y}+B_{z}^{2}\right), \\
u=\frac{1}{2 V}\left(\widehat{B}_{y}^{2}+2{ }_{0} B_{y} \widehat{B}_{y}+B_{z}^{2}\right), \quad \widehat{B}_{y}=B_{y}-{ }_{0} B_{y}, \quad{ }_{0} B_{y}=\sin \varphi .
\end{gathered}
$$

Константы интегрирования выбираются таким образом, чтобы невозмушенное состояние являлось решением получившихся уравнений, которые, следовательно, имеют вид:

$$
\begin{gathered}
\dot{v}=-R_{i} B_{z}-\frac{R_{i} \cos \varphi}{V} n w, \\
\dot{w}=\frac{R_{i} \cos \varphi}{V} n v+R_{i} \widehat{B}_{y}+R_{i} \sin \varphi n\left(n^{-1}-1\right), \\
\dot{\hat{B}}_{y}=R_{e} n w+\frac{R_{e} \cos \varphi}{V} n B_{z}, \quad \dot{B}_{z}=-R_{e} n v-\frac{R_{e} \cos \varphi}{V} n \widehat{B}_{y} .
\end{gathered}
$$

Вблизи $V=V_{\Phi}^{+}$система (4) может быть представлена в форме (1), где $\mathbf{w}=\left(v, w, \widehat{B}_{y}, B_{z}\right)^{t}$,

$$
A=\left(\begin{array}{cccc}
0 & -R_{i} \cos \varphi & 0 & -R_{i} \\
R_{i} \cos \varphi & 0 & R_{i} \cos ^{2} \varphi & 0 \\
0 & R_{e} & 0 & R_{e} \cos \varphi \\
-R_{e} & 0 & -R_{e} \cos \varphi & 0
\end{array}\right)
$$

и с точностью до членов порядка $\left(|\mu \mathbf{w}|+|\mathbf{w}|^{2}\right)$ включительно

$$
F=\left(\begin{array}{c}
\frac{\mu}{2} R_{i} \cos \varphi w-R_{i} \cos \varphi \sin \varphi w \widehat{B}_{y} \\
-\frac{\mu}{2} R_{i} \cos \varphi v+\mu R_{i} \sin ^{2} \varphi \widehat{B}_{y}+\frac{R_{i}}{2} \sin 2 \varphi v \widehat{B}_{y} \\
-\frac{R_{i}}{2} \sin \varphi\left(\widehat{B}_{y}^{2}+B_{z}^{2}\right)-R_{i} \sin ^{3} \varphi \widehat{B}_{y}^{2} \\
-\frac{\mu}{2} R_{e} \cos \varphi B_{z}+R_{e} \cos \varphi \sin \varphi \widehat{B}_{y} B_{z}+R_{e} \sin \varphi w \widehat{B}_{y} \\
\frac{\mu}{2} R_{e} \cos \varphi \widehat{B}_{y}-R_{e} \sin \varphi v \widehat{B}_{y}-R_{e} \cos \varphi \sin \varphi \widehat{B}_{y}^{2}
\end{array}\right) .
$$


Заметим, что правая часть (4) антикоммутирует с изометрией

$$
R=\left(\begin{array}{cccc}
1 & 0 & 0 & 0 \\
0 & -1 & 0 & 0 \\
0 & 0 & 1 & 0 \\
0 & 0 & 0 & -1
\end{array}\right),
$$

т.е. $A R=-R A$ и $F(\mu, R \mathbf{w})=-R F(\mu, \mathbf{w})$. Это означает, что система (4) является обратимой, т.е. среди ее решений находятся решения с четньми $v$ и $\widehat{B}_{y}$ и нечетньми $w$ и $B_{z}$. Такие решения будем назьвать также обратимьми. Собственные значения $\lambda$ матрицы $A$ удовлетворяют уравнению

$$
\lambda^{2}\left[\lambda^{2}-R_{i} R_{e} \cos ^{2} \varphi\left(\operatorname{tg}^{2} \varphi-\left(\sqrt{\frac{R_{e}}{R_{i}}}-\sqrt{\frac{R_{i}}{R_{e}}}\right)^{2}\right)\right]=0 .
$$

В настоящей работе уделено внимание лиш случаю $\varphi>\varphi_{c}$, где $\operatorname{tg}^{2} \varphi_{c}=$ $\left(\sqrt{R_{e} / R_{i}}-\sqrt{R_{i} / R_{e}}\right)^{2}$. По поводу более интересного случая $\varphi<\varphi_{c}$ ограничимся лишь несколькими замечаниями в конце статьи. Критический угол $\varphi_{c}$ разделяет две области (в пространстве параметров) качественно различного поведения ограниченных решений (4) малой амплитуды и так или иначе возникает при выводе модельных уравнений (см., например, [9]). При $\varphi>\varphi_{c}$ уравнение (6) имеет три корня, один из которых (ноль кратности два) лежит на мнимой оси. Заметим, что $|F(\mu, \mathbf{w})| \leqslant c\left(|\mu| \cdot|\mathbf{w}|+|\mathbf{w}|^{2}\right)$ для $\mathbf{w}$ достаточно близких к нулю. Пусть $\mathbf{w}=\mathbf{w}_{0}+\mathbf{w}_{1}$, где $\mathbf{w}_{0} \in E_{0}, \mathbf{a}$ $\mathbf{w}_{1} \in E_{h}$ и, аналогично, для $F_{0}$ и $F_{1}$. Система уравнений (4) имеет вид

$$
\begin{aligned}
& \dot{\mathbf{w}}_{0}=A_{0} \mathbf{w}_{0}+F_{0}\left(\mu, \mathbf{w}_{0}+\mathbf{w}_{1}\right), \\
& \dot{\mathbf{w}}_{1}=A_{1} \mathbf{w}_{1}+F_{1}\left(\mu, \mathbf{w}_{0}+\mathbf{w}_{1}\right),
\end{aligned}
$$

где $A_{0}=\left.A\right|_{E_{0}}, A_{1}=\left.A\right|_{E_{h}}$. Кроме того, очевидно, что сушествуют окрестности нуля $U_{0}^{\prime} \subset E_{0}, U_{1}^{\prime} \subset E_{h}$ и $\Lambda$ точки $\mu=0$ такие, что

$$
F=\left(F_{0}, F_{1}\right)^{t} \in C^{k}\left(\Lambda \times U_{0}^{\prime} \times U_{1}^{\prime}, E_{0} \times E_{h}\right), \quad F(0,0), \partial_{\mathbf{w}} F(0,0)=0 .
$$

Воспользуемся далее следующей теоремой.

ТЕорема 1 (о центральном многообразии [2]). Существуют окрестности нуля $U_{0} \subset U_{0}^{\prime} \subset E_{0}, U_{1} \subset U_{1}^{\prime} \subset E_{h}$, окрестность $\Lambda_{0} \subset \Lambda$ точки $\mu=0$ и функиия

$$
h=h\left(\mu, \mathbf{w}_{0}\right) \in C^{k-1}\left(\Lambda_{0} \times U_{0}, U_{1}\right),
$$

обладающая следующими свойствами:

- множсество

$$
M=\left\{\mathbf{w}_{0}, h\left(\mu, \mathbf{w}_{0}\right) \subset E_{0} \times E_{h} \mid \mathbf{w}_{0} \in U_{0}\right\}
$$


является локальным интегральным многообразием

c $\mu \in \Lambda_{0}$

- каждое решение (7) с $\mu \in \Lambda_{0}, \mathbf{w}_{0}(\xi), \mathbf{w}_{1}(\xi) \in U_{0} \times U_{1}$ для всех $\xi \in \mathbb{R}$ принадлежсит $M$

$-h(0,0)=\partial_{\mathbf{w}_{0}} h(0,0)=0$;

- если $R_{0}: E_{0} \rightarrow E_{0} \quad u \quad R_{1}: E_{h} \rightarrow E_{h}$ являются линейнымми изометриями такими, что $F_{j}\left(\mu, R_{0} \mathbf{w}_{0}, R_{1} \mathbf{w}_{1}\right)=$ $-R_{j} F_{j}\left(\mu, \mathbf{w}_{0}, \mathbf{w}_{1}\right), A_{j} R_{j}=-R_{j} A_{j}, j=0,1, \operatorname{moz} \partial a h\left(\mu, R_{0} \mathbf{w}_{0}\right)=$ $R_{1} h\left(\mu, \mathbf{w}_{1}\right)$.

Из теоремы 1 следует редукция системы (7) для решений, остающихся в $U_{0} \times U_{1}$ для всех $\xi \in \mathbb{R}$. Эти решения удовлетворяют уравнениям

$$
\dot{\mathbf{w}}_{0}=A_{0} \mathbf{w}_{0}+f_{0}\left(\mu, \mathbf{w}_{0}\right)
$$

где $f_{0}\left(\mu, \mathbf{w}_{0}\right)=F_{0}\left(\mu, \mathbf{w}_{0}+h\left(\mu, \mathbf{w}_{0}\right)\right)$. Более того, решения (8) обратимы по отношению к матрице $R_{0}: R=R_{0} \oplus R_{1}, R_{0}: E_{0} \rightarrow E_{0}$. В pacсматриваемом случае $\varphi>\varphi_{c}$ размерность $E_{0}$ (а значит, и центрального многообразия) равна двум. Собственный и присоединенный векторы $A$, соответствующие $\lambda=0$, имеют вид

$$
\phi_{0}=\left(\begin{array}{c}
-\cos \varphi \\
0 \\
1 \\
0
\end{array}\right), \quad \phi_{1}=\left(\begin{array}{c}
0 \\
R_{e}^{-1}-R_{i}^{-1} \cos ^{2} \varphi \\
0 \\
\left(R_{i}^{-1}-R_{e}^{-1}\right) \cos \varphi
\end{array}\right)
$$

$A_{0} \phi_{0}=0, A_{0} \phi_{1}=\phi_{0}, R_{0} \phi_{0}=\phi_{0}, R_{0} \phi_{1}=-\phi_{1}$, а значит,

$$
A_{0}=\left(\begin{array}{ll}
0 & 1 \\
0 & 0
\end{array}\right), \quad R_{0}=\left(\begin{array}{cc}
1 & 0 \\
0 & -1
\end{array}\right)
$$

Для $\mathbf{w}_{0} \in E_{0}$

$$
\mathbf{w}_{0}=\left(\begin{array}{c}
v \\
w \\
\widehat{B}_{y} \\
B_{z}
\end{array}\right)=a_{0} \phi_{0}+a_{1} \phi_{1}
$$

Легко заметить, что $h\left(\mu, \mathbf{w}_{0}\right)=O\left(\mu\left|\mathbf{w}_{0}\right|+\left|\mathbf{w}_{0}\right|^{2}\right)$, а значит, $f_{0}=F_{0}\left(\mu, \mathbf{w}_{0}\right)+$ $o\left(\mu\left|\mathbf{w}_{0}\right|+\left|\mathbf{w}_{0}\right|^{2}\right)$. Таким образом, члены порядка $\mu\left|\mathbf{w}_{0}\right|+\left|\mathbf{w}_{0}\right|^{2}$ могут быть получены, полагая $h=0$.

Оператор, сопряженньй оператору $A$, дается матрищей

$$
A^{*}=\left(\begin{array}{cccc}
0 & R_{i} \cos \varphi & 0 & -R_{e} \\
-R_{i} \cos \varphi & 0 & R_{e} & 0 \\
0 & R_{i} \cos ^{2} \varphi & 0 & -R_{e} \cos \varphi \\
-R_{i} & 0 & R_{e} \cos \varphi & 0
\end{array}\right)
$$


а его присоединенный и собственный векторы имеют вид

$$
\begin{aligned}
& \psi_{0}=R_{i}^{-1} \Delta^{-1}\left(\begin{array}{c}
\left(R_{i}^{-1}-R_{e}^{-1}\right) \cos \varphi \\
0 \\
R_{i} R_{e}^{-1}\left(R_{1}^{-1}-R_{e}^{-1} \cos ^{2} \varphi\right) \\
0
\end{array}\right), \\
& \psi_{1}=R_{i}^{-1} \Delta^{-1}\left(\begin{array}{c}
0 \\
1 \\
0 \\
R_{i} R_{e}^{-1} \cos \varphi
\end{array}\right),
\end{aligned}
$$

где $\Delta=R_{e}^{-1} R_{i}^{-1} \cos ^{2} \varphi\left(\operatorname{tg}^{2} \varphi-\operatorname{tg}^{2} \varphi_{c}\right)$. Уравнение (8) в базисе $\phi_{0}, \phi_{1}$ принимает форму

$$
\dot{\mathbf{a}}=A_{0} \mathbf{a}+g_{0}(\mu, \mathbf{a}),
$$

где $\mathbf{a}=\left(a_{0}, a_{1}\right)^{t}$ и $g_{0}=O\left(\mu|\mathbf{a}|+|\mathbf{a}|^{2}\right)$ имеет компоненты $\left\langle F, \psi_{j}\right\rangle, j=0,1$, $F$-вектор с компонентами $(5)$, а $\langle\cdot, \cdot\rangle$ обозначает обычноескалярноепроизведение в $\mathbb{R}^{4}$. С точностью до членов второго порядка малости

$$
\begin{aligned}
& \left\langle F, \psi_{0}\right\rangle=\Delta^{-1}\left(\mu r_{1} a_{1}+r_{2} a_{0} a_{1}\right), \\
& \left\langle F, \psi_{1}\right\rangle=\Delta^{-1}\left(\mu a_{0}-\frac{3}{2} \sin \varphi a_{0}^{2}-\frac{1}{2} r_{3} a_{1}^{2}\right),
\end{aligned}
$$

где

$$
\begin{aligned}
& r_{1}=-\frac{1}{2} \cos ^{2} \varphi\left(1+\cos ^{2} \varphi\right)\left(R_{e}^{-1}-R_{i}^{-1}\right)^{2}, \\
& r_{2}=R_{e}^{-1} R_{i}^{-1} \sin \varphi \cos ^{4} \varphi\left(\operatorname{tg}^{4} \varphi+\operatorname{tg}^{2} \varphi_{c}\right), \\
& r_{3}=\left(R_{e}^{-1}-R_{i}^{-1}\right)^{2} \cos ^{2} \varphi \sin \varphi .
\end{aligned}
$$

Уравнения (9), таким образом, принимают вид

$$
\begin{aligned}
& \dot{a}_{0}=a_{1}+\Delta^{-1}\left(\mu r_{1} a_{1}+r_{2} a_{0} a_{1}\right)+o\left(\mu|\mathbf{a}|+|\mathbf{a}|^{2}\right), \\
& \dot{a}_{1}=\Delta^{-1}\left(\mu a_{0}-\frac{3}{2} \sin \varphi a_{0}^{2}-\frac{1}{2} r_{3} a_{1}^{2}\right)+o\left(\mu|\mathbf{a}|+|\mathbf{a}|^{2}\right) .
\end{aligned}
$$

2. Квази-нормальная форма уравнения (10) (с точностью до членов порядка 2 включительно) имеет вид [2]

$$
\dot{\alpha}_{0}=\alpha_{1}, \quad \dot{\alpha}_{1}=\frac{1}{\Delta}\left(\mu \alpha_{0}-\frac{3}{2} \sin \varphi \alpha_{0}^{2}\right) .
$$

Производя масштабные преобразования $\alpha_{0}=\mu \sin ^{-1} \varphi \beta_{0}(y), \alpha_{1}=\mu \nu \beta_{1}(y)$, $y=\nu \xi, \nu=|\mu|^{1 / 2} \Delta^{-1 / 2}$, получим

$$
\partial_{y} \beta_{0}=\beta_{1}, \quad \partial_{y} \beta_{1}=\operatorname{sgn}(\mu)\left(\beta_{0}-\frac{3}{2} \beta_{0}^{2}\right) .
$$

Уравнения (11) для $\mu>0$ имеют решения следующих типов:

i) уединенные волны $(H=0): \beta_{0}=c h^{-2} y / 2$;

ii) периодические волны. 
Для $-4 / 27<h<0$ (где $H=\operatorname{sgn} \mu \mu^{2} \nu^{2} \sin ^{-2} \varphi h$ ) решениями (11) являются периодические орбиты, параметризованные периодом и фазовым сдВигом.

Для $\mu<0$ уравнения (11) также обладают периодическими решениями (когда $0<h<4 / 27$ ).

3. Для потока на центральном многообразии (10) имеет место факт грубости солитоноподобных решений относительно возмушений высшего порядка по амплитуде. Доказательство соответствующего утверждения, сформулированного ниже в виде теоремы 2, дано, например, в [6], где система, аналогичная (10), возникает при рассмотрении капиллярно-гравитационных волн на поверхности идеальной жидкости конечной глубины. В настоящей работе приводится более простое доказательство теоремы 2 .

ТЕорема 2 (о грубости солитоноподобных решений). Предположим $\boldsymbol{\alpha}^{*}=\left(\alpha_{0}^{*}, \alpha_{1}^{*}\right)$ соответствует солитоноподобному решению уравнений (11). Тогда для достаточно малых $\mu_{0}, \mu \in\left(0, \mu_{0}\right)$, существует семейство солитоноподобных решений $\mathbf{a}=\left(a_{0}, a_{1}\right)(\mu)$ полной системы (10). Справедливы оценки

$$
\left|\mathbf{a}-\boldsymbol{\alpha}^{*}\right| \leqslant c_{0} \mu^{2} \exp (-\sigma|y|),
$$

где с с зависит только от $\mu_{0}, \sigma<1$.

ДоКАЗАТЕЛЬСТво. Обратимость уравнений (10) по отношению к $R_{0}$ означает, что мы можем ограничиться рассмотрением лишш таких решений, у которых $a_{0}$ (или $\alpha_{0}$ ) - четная функция. Запишем уравнения (10) в виде

$$
\begin{aligned}
& \dot{\alpha}_{0}=\alpha_{1}+r_{0}\left(\mu, \alpha_{0}, \alpha_{1}\right), \\
& \dot{\alpha}_{1}=\Delta^{-1}\left(\mu \alpha_{0}-\frac{3}{2} \sin \varphi \alpha_{0}^{2}\right)+r_{1}\left(\mu, \alpha_{0}, \alpha_{1}\right),
\end{aligned}
$$

где $\left|r_{0}\right| \leqslant c|\mu|^{s}$ для любого $s$ и нечетна по $\alpha_{1}$ (в свете сказанного выше нечетна по $y$ ), а $r_{1}\left(\mu, \alpha_{0}, \alpha_{1}\right)=O\left(\mu^{2}\right)$ четна по $\alpha_{1}$ (а значит, по $y$ ). Пользуясь изложенными свойствами, $r_{0}$ и $r_{1}$ мы можем положить $r_{0}=\alpha_{1} \widehat{r}_{0}$ и заменить $\alpha_{1}$ на $\alpha_{1}\left(1+\widehat{r}_{0}\right)$. Очевидно, это эквивалентно $r_{0}=0$ (при этом упомянутая замена дает вклад в $r_{1}$, сохраняющий его четность). Уравнения (12), таким образом, после масштабного преобразования могут быть переписаны в виде

$$
F=\ddot{\beta}_{0}-\beta_{0}+\frac{3}{2} \beta_{0}^{2}+r\left(\mu, \beta_{0}, \dot{\beta}_{0}\right)=0,
$$

где $r$-по-прежнему четная функция от $y$. Определим далее банаховы пространства

$$
\begin{gathered}
C_{\sigma, j}^{e}=\left\{\beta_{0} \in C^{j}(\mathbb{R}), \sup _{y} \exp (\sigma|y|)\left|\beta_{0}^{(m)}\right|<\infty, m \leqslant j, \beta_{0}(y)=\beta_{0}(-y)\right\} \\
j=0,1,2 .
\end{gathered}
$$


Очевидно, что $\beta_{0}^{*}=\operatorname{ch}^{-2}(y / 2)$ принадлежит $C_{1,2}^{e}$. Пусть $F: C_{\sigma, 2}^{e} \rightarrow C_{\sigma, 0}^{e}$, где $\sigma<1$. Из теоремы о неявной функции будет следовать существование и единственность $\beta_{0}(\mu) \in C_{\sigma, 2}^{e}$, для $\mu \in\left(0, \mu_{0}\right]$, удовлетворяющего уравнению (13), если оператор

$$
L=\frac{\partial F}{\partial \beta_{0}}\left(0, \beta_{0}^{*}\right): C_{\sigma, 2}^{e} \rightarrow C_{\sigma, 0}^{e}
$$

имеет ограниченный обратный. Доказательство первой части утверждения теоремы, таким образом, сводится к проверке этого факта. Легко видеть, что

$$
L=\frac{d^{2}}{d x^{2}}-1+3 \beta_{0}^{*}
$$

и обратимость $L$ эквивалентна существованию и единственности решения уравнения

$$
L u=g
$$

для любого $g \in C_{\sigma, 0}^{e}$. Последнее можно усмотреть из следующих соображений.

i) Однородное уравнение $L u=0$ не имеет решений в $C_{\sigma, 2}^{e}$. В самом деле, решение $u_{1}=\partial_{y} \beta_{0}^{*}$ - нечетная функция, а линейно независимое с ним $u_{2}=a u_{1}+b u_{1} \int u_{1}^{-2}(y) d y-$ четная (если константы $a$ и $b$ выбираются из условий $\left.u_{2}(0)=1, u_{2}^{\prime}=0\right)$, но возрастающая функция. Следовательно, решение (14) единственно, если существует.

ii) Искомое решение (14) дается формулой

$$
u=u_{2} \int_{y}^{\infty} u_{1} g d y+u_{1} \int_{0}^{y} u_{2} g d y .
$$

Кроме того, из (15) следует, что $\|u\|_{C_{\sigma, 2}^{e}} \leqslant C\|g\|_{C_{\sigma, 0}^{e}}$.

Первая часть утверждения теоремы 2 , таким образом, доказана. Запишем далее решение $\beta_{0}$ уравнения (12) в виде $\beta_{0}=\beta_{0}^{*}+\widehat{\beta}_{0} . \widehat{\beta}_{0}$ удовлетворяют уравнению $L \widehat{\beta}_{0}=G\left(\mu, \widehat{\beta}_{0}\right)$, где $G=O\left(\mu, \widehat{\beta}_{0}^{2}\right)$ - четная функция $y$, a, значит, $\left\|\widehat{\beta}_{0}\right\|_{C_{\sigma, 2}^{e}} \leqslant\left\|L^{-1}\right\| \cdot\left\|G\left(\mu, \widehat{\beta}_{0}\right)\right\|_{C_{\sigma, 0}^{e}}$, откуда немедленно следует второе утверждение теоремы.

В заключение отметим, что в случае $\varphi<\varphi_{c}$ система (4) обладает более многочисленным набором решений, среди которых находятся быстроосциллирующие периодические волны (рябь), периодические волны, скомбинированные из медленно- и быстроосциллируюших волн, квазипериодические волны, а также уединенные волны (гомоклинические решения) с незатухающими быстроосциллирующими хвостами. Подробньй анализ 
этого случая, а также альфвеновской ветви $\left(V_{\Phi}^{-}=\cos \varphi\right)$ будет опубликован.

Математический институт

Поступило

им. В.А. Стеклова РАН

17.05 .94

Исправленный вариант

10.06 .95

\section{СПИСОК ЦИТИРОВАННОЙ ЛИТЕРАТУРЫ}

[1] Плисс В.А. Принцип редукции в теории устойчивости движения // Изв. АН CCCP. Сер. матем. 1964. T. 28. C. 1297-1324.

[2] Iooss G., Adelmeyer M. Topics in bifurcation theory and applications. World Scientific, 1992.

[3] Kirchgässner K. Wave solutions of reversible systems and applications // J. Diff. Equat. 1982. V. 45. P. 113-127.

[4] Mielke A. Reduction of quasilinear elliptic equations in cylindrical domains with applications // Math. Meth. Appl. Sci. 1988. V. 10. P. 51-66.

[5] Mielke A. Normal hyperbolicity of center manifolds and Saint-Venant's principle // Arch. Rat. Mech. Anal. 1990. V. 110. P. 353-372.

[6] Iooss G., Kirchgässner K. Water waves for small surface tension: an approach via normal form // Proc. Royal Soc. Edinburgh. 1992. V. 122A. P. 267-299.

[7] Saffman P. G. On hydromagnetic waves of finite amplitude in a cold plasma // J. Fluid Mech. 1961. V. 11. P. 552-566.

[8] Kakutani T., Ono H., Tanuiti T., Wei C.-C. Reductive perturbation method in nonlinear wave propagation. II. Application to hydromagnetic waves in cold plasma // J. Phys. Soc. Japan. 1968. V. 24. P. 1159-1166.

[9] Kakutani T., Ono H. Weak non-linear hydromagnetic waves in a cold collision-free plasma // J. Phys. Soc. Japan. 1969. V. 26. P. 1305-1318.

[10] Ильичев А.Т. О существовании семейства солитоноподобных решений уравнения Кавахары // Матем. заметки. 1992. Т. 52. С. 42-50.

[11] Il'ichev A. T., Semenov A. Yu. Stability of solitary waves in dispersive media described by a fifth order evolution equation // Theoret. Comput. Fluid Dynamics. 1992. V. 3. P. 307-326.

[12] Маслов В. П., Омельянов Г. А. Взаимодействие коротких волн в слабо дисперсионной плазме. I // Укр. матем. ж. 1987. Т. 39. С. 464-472. 